\title{
LA OPINIÓN DE NUESTRO ALUMNADO SOBRE LOS MÉTODOS DE ENSEÑANZA Y SISTEMAS DE EVALUACIÓN EMPLEADOS EN LA ESPECIALIDAD DE EDUCACIÓN FÍSICA DE LOS ESTUDIOS DE MAGISTERIO
}

\author{
Edmundo Loza Olave \\ Universidad de La Rioja
}

\begin{abstract}
RESUMEN. Este trabajo pretende, mediante la elaboración de un cuestionario anónimo pasado a los propios alumnos de la Especialidad de Educación Fisica, llevar a cabo un estudio sobre los métodos de enseñanza y los sistemas de evaluación que utiliza el profesorado de las E.U.M. y Facultades de Educación en las que se imparte la citada Especialidad dentro de los estudios de Magisterio.

Esto nos va a permitir conocer algo más acerca de la realidad de nuestros centros y las demandas e intereses de nuestro alumnado respecto a los estudios que cursan y saber qué es lo que éstos opinan sobre el proceso de enseñanza-aprendizaje que en sus centros de formación se sigue.

Para ello se realiza una investigación, que podriamos insertar en el ámbito de las investigaciones educativas, cuyo objetivo, además de ofrecer información sobre una determinada situación educativa, trata de tener en cuenta los diferentes aspectos implicados en la misma, por lo que su dimensión fundamental es de carácter descriptivo, aunque posteriormente se lleven a cabo valoraciones y propuestas de corte más evaluativo.
\end{abstract}

ABSTRACT.A This text tries to carry out, through an anonymous questionnaire answered by the students of Physical Education, a study of the teachinh methodology and teacher evaluation systems used by the teachers in the Teacher Training Schoo's and Educational Faculties. In order to know not only the demands and interests of our students, but their opinion of the teaching-learning process carried out in the Teacher Training Schools.

To get this aim we have done a research, that it can be inserted in the current educational investigation trends, whose purpose is to offer information about a given educational situation. This research also tries to take into account the different aspects involved, so that its fundamental dimension is descriptive, but afterwards it will also allow to carry out valuations and proposals of evaluation

\section{Introducción}

Dentro del análisis de la situación actual de los estudios de Magisterio en la Especialidad de Educación Física, consideramos que es un asunto que cobra especial 
importancia a la hora de plantearnos una conexión lo más estrecha posible entre nuestras intenciones educativas, la realidad de nuestros centros y las demandas e intereses de nuestros alumnos respecto a los estudios que cursan, conocer qué es lo que éstos opinan sobre el proceso de enseñanza-aprendizaje que en sus centros de formación se sigue.

Investigar los métodos de enseñanza y los sistemas de evaluación que utiliza el profesorado de las E.U.M. supone averiguar, indirectamente, si éste está implicado en el que creemos constituye el fin mismo de dichos centros: dotar a los futuros maestros de los útiles y planteamientos metodológicos más acordes para "aprender a enseñar", ya que entendemos que elementos como la metodología, constituyen de alguna manera, uno de los principales puntos de apoyo en los que se sustentará todo el proceso de enseñar y aprender, mientras que la evaluación por su parte, es una de las claves de cualquier sistema didáctico que se utilice en el aula y determina en gran medida al resto de los componentes que integran la estructura de cualquier modelo didáctico-educativo.

Con tal fin, se realiza una investigación, que podríamos insertar en el ámbito de las investigaciones educativas, cuyo objetivo es ofrecer información sobre una determinada situación educativa, tratando de tener en cuenta los diferentes aspectos implicados en la misma, por lo que su dimensión fundamental es de carácter descriptivo, aunque posteriormente se lleven a cabo valoraciones y propuestas de corte algo más evaluativo.

En el fondo, lo que subyace es una inquietud por la renovación, mejora y puesta al día de todo y todos los que, de un modo u otro, nos hallamos implicados en la formación de nuestro alumnado, pudiéndose considerar este trabajo igualmente, como un ejercicio de sana autocrítica.

Para todo ello, hemos partido de la elaboración de un cuestionario anónimo (previamente validado y del que únicamente mostramos algunos de sus ítems más significativos), que nos va a permitir acercarnos lo más posible a la realidad actual de nuestros centros de enseñanza.

El citado cuestionario, recoge la opinión de alumnos de la Especialidad de Educación Física de todas las Escuelas Universitarias de Magisterio y/o Facultades de Educación de nuestro país en las que se imparten dichos estudios, con lo que la información que se obtenga, nace en y desde los propios centros, ya que parece más apropiado que las opiniones que al respecto se analicen, provengan de aquellos que directamente viven, y por tanto conocen, los avatares propios de las citadas instituciones.

El cuestionario, ha sido pasado a una muestra aleatoria de 908 alumnos de la Especialidad de Educación Física (cifra que supone el 10\% de la totalidad de estudiantes de dicha Especialidad en nuestro país), de todos los centros de España en los que se imparte ésta, siendo éstos un total de 44 . Una vez recogidas y revisadas todas las encuestas, se procedió a la grabación de los datos en soporte informático y a la verificación de los mismos, con el fin de llevar a cabo la investigación, mediante un estudio tanto cuantitativo como cualitativo de la información recogida, con una parte del paquete informático de los programas estadísticos SPSS. 


\section{Breve aproximación teórica y conceptual}

Consideramos que la Metodología es un apartado del proceso enseñanza-aprendizaje, que hace referencia a todos los aspectos relacionados con los caminos elegidos para conseguir los objetivos señalados y que constituye la totalidad de momentos, situaciones y técnicas de aprendizaje, coordinados de forma lógica con el fin de encauzar dicho aprendizaje hacia unos objetivos concretos, ya determinados.

Creemos que no existe una metodología que pueda ser considerada como ideal, sino que existen "metodologías adecuadas o no", en virtud del momento, del entorno, de la tarea y de un sin fin de variables, que habrán de tenerse en cuenta y que determinarán si la aplicación de una metodología concreta ha resultado oportuna.

Obsérvese, de todos modos, que todo esto se refiere al caso en el que exista una intencionalidad de enseñar/aprender, puesto que en el caso contrario, el del azar, no existe experiencia de enseñanza-aprendizaje y por tanto tampoco metodología aplicada, ya que además de intencional, la metodología debe ser sistemática, pues debe derivar de la aplicación de una estrategia pedagógica suficientemente justificada. Esto significa que la utilización aislada de métodos sin tener en cuenta el proceso en el que se hallan inmersos, no debe considerarse metodología, o correcta aplicación metodológica si se quiere ser más minucioso, puesto que el carácter intencional del que se hablaba anteriormente también afecta a la elección metodológica, y esta elección consecuentemente, debe respetar un plan de conjunto que vendrá diseñado desde las grandes líneas pedagógicas, hasta la puesta en práctica de la acción docente, pasando por las orientaciones departamentales o las definiciones en el plan anual, en el bloque temático, etc.

Por su parte el concepto didáctico "Evaluación" fue uno de los que más sufrió las restricciones positivistas hace no muchos años. Se redujo de tal manera su significado, que para la mayoría de los educadores, era (y en ocasiones todavía sigue siéndolo), simplemente el proceso de medida de la adquisición de conocimientos por parte de los alumnos.

En los setenta surgió un destacado y renovado interés por el aspecto cualitativo de la evaluación, considerándola como una actividad procesual encargada de la observación, análisis y toma de decisiones acerca del funcionamiento y evolución de la actividad docente en la clase, como medio para conocer y adaptar mejor, tanto alumno como profesor, la formación a las necesidades. De esta manera, la evaluación no se limita a resolver la normativa académica que exige una calificación indicativa de las capacidades del alumno o de su nivel de conocimientos, sino que va a ayudar tanto al alumno como al profesor a conocer los resultados durante y después del aprendizaje, así como la validez y competencia del conjunto de factores que integran el complejo mecanismo de la enseñanza.

Creemos que la evaluación juega un papel comprometido con la totalidad del proceso educativo, formando parte de un esquema global, que incluye desde el análisis de las situaciones previas, generales y específicas, hasta el seguimiento de la ejecución del proceso, pasando por la programación de las tareas a realizar; pretendiendo, en definitiva, guiar las diferentes decisiones, que en cada uno de estos apartados se deben ir tomando. 
En definitiva, podriamos afirmar que la evaluación es una de las claves del arco sistémico de la educación; es decir, del conjunto de elementos estructurados y organizados (contenidos, objetivos, métodos, recursos, espacio, etc.), que buscan lograr un mismo objetivo. Se puede convenir en que la evaluación es una actividad procesual, y por tanto dinámica ("retroalimentadora" si se quiere más específicamente), en el sentido de que ofrece información que abastece internamente al propio proceso didáctico, permitiéndole a éste, detectar los aciertos y los errores, determinar las causas y proponer las soluciones.

Finalmente, entendemos igualmente que la evaluación ha de contemplarse como un proceso de investigación sobre la práctica, y por tanto estimulará hacia un conocimiento permanente y revisable sobre dicha práctica profesional.

\section{Análisis y resultados}

3. 1. Formas de enseñanza-aprendizaje utilizadas preferentemente en nuestros centros

Con el fin conocer la opinión de los alumnos sobre lo tratado en la introducción de este estudio, hemos de comentar que en primer lugar se les planteó que expusieran qué formas de enseñanza-aprendizaje son las que ha utilizado preferentemente el profesorado para desarrollar los contenidos de sus materias, estableciendo para ello tres niveles de frecuencia con el fin de que indiquen en cuál de ellos han de ubicarse las distintas formas de enseñanza-aprendizaje planteadas. Estos niveles citados son "Muy utilizado", "Utilización media" y "Poco utilizado",

Por su parte las formas de enseñanza-aprendizaje planteadas son las siguientes:

1. Lección magistral o exposición de la materia por el profesor

2. Clases teórico-prácticas

3. Seminarios

4. Clase impartida por el alumno

Veamos la distribución de los resultados de esta primera cuestión.

\section{Formas}

1. Lección magistral

2. Clases teórico-prácticas

3. Seminarios

4. Estudio de casos

5. Grupos de discusión

6. Clases prácticas

7. Clase impartida alumno
Muy utilizado\%

70,2
35,6
1,0
0,6
5,3
29,9
8,6

5. Grupos de discusión

6. Clases prácticas

7. Estudio de casos

Una vez analizados los datos recogidos, puede afirmarse que la forma más utilizada es la "lección magistral" ya que alcanza el mayor porcentaje de respuestas (un $70,2 \%)$ del nivel "muy utilizado". A continuación aparecen las "clases teórico-prácti-

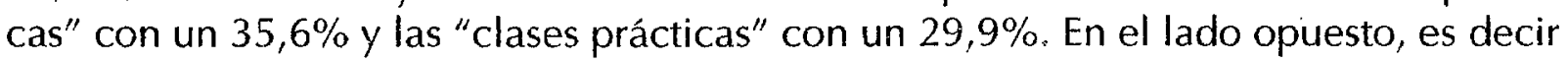
en el de los porcentajes de formas "poco utilizadas", son los "seminarios", el "estudio 
de casos", los "grupos de discusión" y la "lección impartida por el alumno" los que alcanzan los valores más altos.

Esta distribución de los porcentajes, confirma lo que ya la simple experiencia nos dice: la lección magistral; la simple exposición de información en la que el docente emplea la mayor parte de su tiempo en transmitir unos conocimientos y el discente prácticamente reduce su pasiva actividad a tomar apuntes para posteriormente devolverlos en forma de examen, sigue siendo la fórmula más frecuentemente empleada.

Por el contrario aquellas técnicas consideradas las opuestas a la lección magistral como los seminarios, el estudio de casos y los grupos de discusión (en las cuales el alumno se convierte en un ser activo y protagonista), siguen siendo las menos frecuentemente utilizadas según indican nuestros alumnos.

No podemos pasar por alto el hecho de que en unos estudios como los nuestros, en los que se supone que las clases prácticas han de ocupar un lugar importante en el desarrollo de los mismos, estas no han logrado el primer lugar en esta valoración, lo cual debería llevarnos a la oportuna reflexión al respecto

\section{2. Opinión sobre las diferentes formas de enseñanza-aprendizaje empleadas}

Nos interesa saber ahora qué opinión les merecen a los alumnos, las anteriormente citadas formas de enseñanza-aprendizaje. Para ello les hemos pedido, que manifiesten el grado de aceptación o valoración que conceden a las distintas formas de enseñanza-aprendizaje ya vistas. Al respecto, hemos establecido nuevamente tres niveles, en este caso de adecuación, por los que los encuestados se pueden decantar. Estos tres niveles son: "muy adecuado", "adecuado" y "poco adecuado".

Este epígrafe nos va a permitir conocer el grado de aceptación o rechazo de la realidad actual así como el ánimo innovador o interés de cambiar hacia otras opciones más acordes con las tendencias educativas actuales.

Veamos nuevamente la distribución de los porcentajes de las respuestas del alumnado.

\section{Formas}

Lección magistral

Clases teórico-práct.

Seminarios

Estudio de casos

Grupos de discusión

Clases prácticas

Clase impartida alumno
Muy adecuado \%

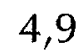

66,4

21,0

30,2

52,6

90,6

40,6

$\begin{array}{cr}\text { Adecuado } \% & \text { Poco ade } \\ 41,8 & 53,3 \\ 33,2 & 0,5 \\ 60,1 & 18,9 \\ 55,1 & 14,7 \\ 43,0 & 4,4 \\ 9,1 & 0,3 \\ 46,8 & 12,6\end{array}$

Parece evidente, a juzgar por la distribución de los porcentajes observada, que las "clases prácticas" es la opción metodológica que mayor aceptación tiene entre el alumnado ya que casi la totalidad de este colectivo se decanta por ella como forma "muy adecuada" de enseñanza-aprendizaje.

Le siguen "las clases teórico-prácticas (más de la mitad del alumnado las considera muy adecuadas), y los "grupos de discusión" considerados igualmente por la mitad 
de nuestros alumnos como sistema de trabajo muy adecuado, así como la "clase impartida por un alumno".

En el extremo opuesto, se halla la "lección magistral", que es considerada por algo más de la mitad del alumnado como alternativa "poco adecuada" o de escaso valor pedagógico con lo que alcanza la consideración de opción menos aceptada por el citado colectivo, del que únicamente un bajísimo 4,9\% la considera como "muy adecuada", aunque también aparece con considerable porcentaje de respuestas en "adecuada". Así pues, podemos decir que, aunque el porcentaje del alumnado que consideran a la lección magistral como una alternativa poco adecuada es el más alto de las tres opciones, no se puede afirmar taxativamente que el rechazo de ésta sea claro, ya que pese a que el entusiasmo por la misma evidentemente no es grande, las posiciones intermedias si que alcanzan porcentajes considerables.

Por su parte, el resto de las alternativas como "seminarios" y "estudios de casos" muestran unos porcentajes en los que la posición intermedia de "adecuado", es la más importante por lo puede pensarse que estos no son ni especialmente valorados ni rechazados, principalmente en el caso de los seminarios, donde el nivel porcentaje "adecuado", alcanza el 60,1\% de las respuestas.

\section{3. La opinión del alumnado en el uso de los distintos métodos para desarrollar las} asignaturas

Bajo este epígrafe pretendemos conocer si los profesores tienen en cuenta la opinión del alumnado sobre los métodos más adecuados para desarrollar las distintas asignaturas de sus estudios o, dicho de otra manera, cuál es la participación de nuestros alumnos en el proceso de enseñanza-aprendizaje que tiene lugar en nuestros centros. Esta cuestión le ha sido planteada al alumnado y sus respuestas han sido las siguientes.

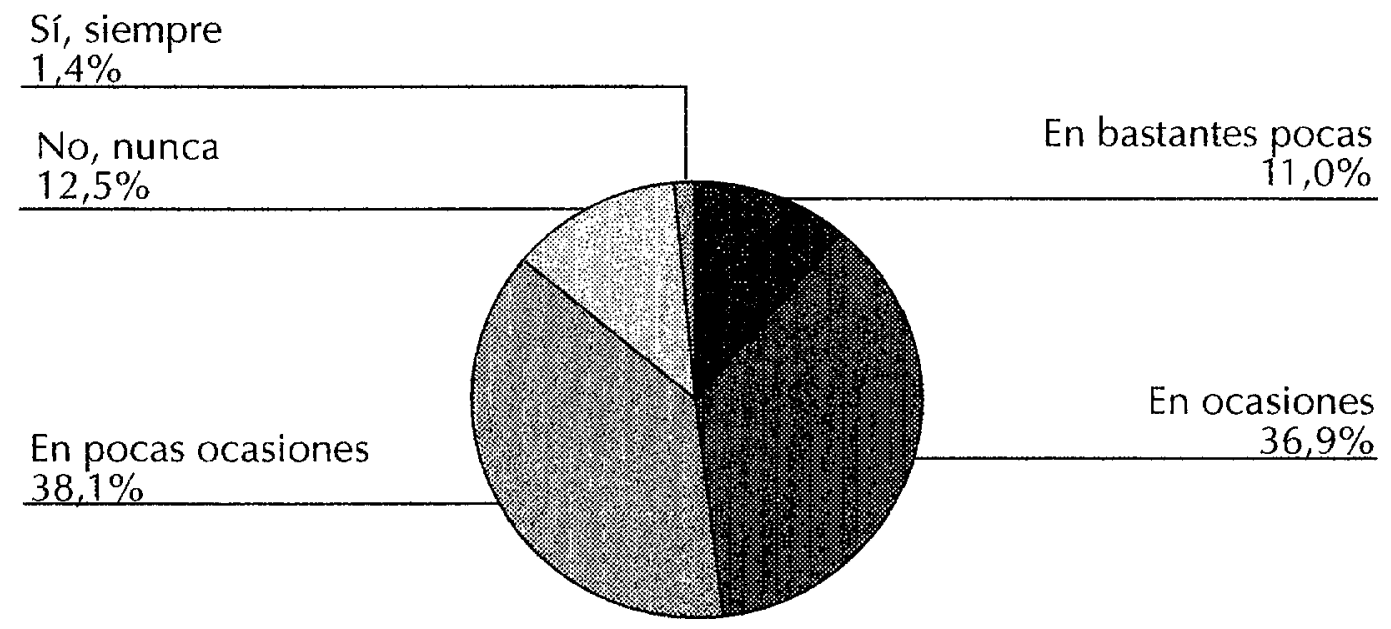

Gráfico $n^{\circ}$. 1. La opinión del alumnado en el uso de los distintos métodos para desarrollar las asignaturas

Según indican nuestros alumnos, el profesorado de nuestros centros no cuenta asiduamente con la opinión de éstos en el desarrollo de sus asignaturas, ya que si suma- 
mos las dos primeras opciones de respuesta que pueden darse, únicamente un reducido $12,4 \%$ de las mismas indican que esto sucede con frecuencia, mientras que si sumamos las opciones de respuesta cuarta y quinta, un importante 50,6\% de ellas muestran que esta práctica no es habitual e incluso en un $12,5 \%$ de respuestas, se señala que esto no sucede nunca.

\section{4. Grado de aceptación de algunas experiencias de enseñanza-aprendizaje}

En este apartado pretendemos ver qué opina nuestro alumnado sobre algunas experiencias de enseñanza-aprendizaje concretas. La relación de experiencias de aprendizaje o técnicas de trabajo seleccionadas es la siguiente:

1 El estudio independiente

2 Trabajos monográficos

3 Recesiones bibliográficas

Para las respuestas de nuestros encuestados, hemos establecido tres niveles, nuevamente de adecuación, por los que éstos, , se pueden decantar. Estos tres niveles y sus respuestas son las siguientes: "muy adecuado", "adecuado" y "poco adecuado".

\section{Técnicas}

Estudio independiente

Trabajos monográficos

Recesiones bibliográf.

Crítica de textos

Trabajos de investigac.
Muy adec. $\%$

23,8

14,3

11,0

25,8

70,8
4 Crítica de textos

5 Trabajos de investigación

Entre las respuestas del alumnado los "trabajos de investigación" aparecen como la técnica de trabajo que el alumnado considera "muy adecuada" en un más alto porcentaje de respuestas, ya que casi las tres cuartas partes del mismo, así lo manifiesta. Parece pues claro que es esta opción la que más positivamente valoran nuestros alumnos.

El resto de las opciones formuladas, no reciben porcentajes ni de rechazo manifiesto ni de aceptación clara, siendo los porcentajes de aceptación intermedia los que alcanzan mayores cotas. Únicamente las recesiones bibliográficas, con casi un 40,0\% de respuestas de "poco adecuado", parece que no cuentan con la estima del alumnado.

\section{5. Sistemas de evaluación utilizados preferentemente en nuestros centros}

En primer lugar, nos interesa conocer cuáles son los sistemas de evaluación que preferentemente utilizan los profesores de nuestros centros. Para ello, hemos formulado a nuestros alumnos una pregunta de idéntica estructura a la formulada anteriormente ("Formas de enseñanza-aprendizaje utilizadas preferentemente en nuestros centros").

Las posibles opciones de respuesta eran las siguientes:

1 Examen escrito sobre uno o más temas

4 Trabajos individuales

2 Pruebas objetivas (tipo test)

3 Exámenes orales

5 Trabajos colectivos

6 Supuestos prácticos 
La distribución de los porcentajes de lo respondido por nuestros alumnos es esta.

\section{Sistemas}

Examen escrito

Pruebas objetivas

Exámenes orales

Trabajos individuales

Trabajos colectivos

Supuestos prácticos
Muy utilizado \%

$$
\begin{array}{r}
90,5 \\
36,2 \\
0,7 \\
36,0 \\
44,0 \\
9,8
\end{array}
$$

Utilizac. media\% Poco Utilizado\%

$\begin{array}{rr}8,0 & 1,5 \\ 39,1 & 24,6 \\ 6,2 & 92,8 \\ 46,8 & 17,1 \\ 44,4 & 11,5 \\ 35,8 & 54,4\end{array}$

Los resultados que se desprenden de esta cuestión, muestran que no hay duda de que el examen escrito constituye la fórmula mayoritariamente utilizada como sistema de evaluación, ya que casi la totalidad de nuestros alumnos afirman que es "muy utilizado". Le siguen, aunque ya con una gran diferencia en la distribución de los porcentajes, los "trabajos colectivos" y los "trabajos individuales".

En el lado opuesto, se hallan los "supuestos prácticos" con un escaso $9,8 \%$ de respuestas "muy utilizado" y un importante 54,4\% de "poco utilizado" y especialmente los "exámenes orales", que con 92,8\% de respuestas "poco utilizado", queda claro que es la fórmula menos utilizada. Al respecto cabría indicar que nos llama la atención el hecho de que siendo los supuestos prácticos uno de los elementos más importantes de las oposiciones al cuerpo de profesores de E. F. en Primaria, sea tan escasamente utilizado en nuestros centros. Ciertamente es un desfase sobre el que quizá habría que reflexionar.

Por último, la fórmula "pruebas objetivas" arroja unos resultados en los que el equilibrio entre las tres posibles opciones de respuesta es prácticamente total, por lo que cabe pensar que su uso no es especialmente utilizado ni tampoco especialmente descartado.

\section{6. Grado de aceptación u opinión de los sistemas de evaluación empleados}

Conozcamos ahora qué grado de aceptación u opinión le merecen las distintas herramientas de evaluación a nuestro alumnado.

$\begin{array}{lccc}\text { Sistemas } & \text { Muy adecuado\% } & \text { Adecuado\% } & \text { Poco adecuado\% } \\ \text { Examen escrito } & 12,3 & 64,8 & 23,0 \\ \text { Pruebas objetivas } & 18,3 & 55,3 & 26,3 \\ \text { Exámenes orales } & 14,7 & 37,0 & 48,2 \\ \text { Trabajos individuales } & 34,4 & 56,0 & 9,6 \\ \text { Trabajos colectivos } & 42,0 & 45,3 & 12,7 \\ \text { Supuestos prácticos } & 56,0 & 36,5 & 7,5\end{array}$

La alternativa que cuenta con mayor aceptación entre nuestros alumnos, es los "supuestos prácticos" ya que en la columna correspondiente al nivel de "muy adecuado" los mayores porcentajes los logra esta opción.

Tras los supuestos prácticos, aparecen los "trabajos colectivos" y los "trabajos individuales", con un $42,0 \%$ y un $34,4 \%$ de respuestas "muy adecuado" respectivamen- 
te, aunque ya con valores superiores de la opción intermedia en ambos casos, por to que quizá convenga considerar que el nivel de aceptación de estos, no está muy claro que sea especialmente elevado.

Del resto de las opciones formuladas, destaca como poco aceptada la de los "exámenes orales" ya que así la califican el alumnado con casi un 50,0\% de las respuestas computadas. Este dato, al compararlo nuevamente con el que veíamos en el epígrafe anterior, en el que el examen oral aparecía como el sistema de evaluación menos utilizado por el profesorado de nuestros centros, muestra, a diferencia de lo ocurrido en dicho caso anterior con los supuestos prácticos, cierta sintonía entre los deseos del alumnado y la tendencia que se sigue en nuestros centros.

Por su parte el "examen escrito", que en el epígrafe anterior (dedicado a conocer los sistemas de evaluación más utilizados), aparecía como el más utilizado, en éste aparece considerado como poco adecuado por casi una cuarta parte del alumnado, con lo que nuevamente puede hablarse de cierto desfase entre lo que el alumnado se encuentra en sus centros y lo que realmente parece demandar.

\section{7. Valoración del tipo de exámenes empleado en nuestros centros}

Una vez asumido que el examen, pese a su baja consideración, se utiliza como herramienta de evaluación de forma generalizada, queremos conocer a continuación cómo valoran nuestros alumnos el tipo de exámenes que realizan en nuestros centros docentes.

Para ello, les hemos formulado una propuesta a través de la cual ellos juzgan el tipo de exámenes que realizan mediante siete dimensiones bipolares.

El polo izquierdo (A), indica una nota negativa de los procedimientos de evaluación, mientras que el polo de la derecha (B), los considera positivos. El alumnado puede optar, para responder cada uno de estos dos polos, por tres posibles opciones que

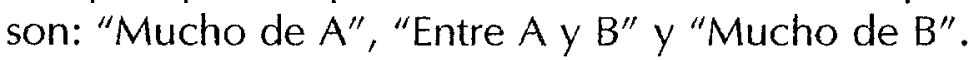

A continuación vamos a ver los dos polos comentados y sus respectivos apartados.

\section{POLO A}

1. Son exámenes que fomentan la memorización

2. En los exámenes nos piden teoría

3. Olvido rápidamente el contenido de lo que me examino

4. Durante el examen el profesor se muestra especialmente riguroso, distanciado...

5. Nos examinan del contenido de un solo libro

6 La nota que da el profesor es inamovible una modificación

7 No me explican la calificación que me dan

\section{POLO B}

1. Fomentan la elaboración personal y la creatividad

2. En los exámenes piden aplicaciones prácticas

3. Conservo bastante el contenido de lo que me examino

4. Durante el examen el profesor se, muestra igual que en clase normal

5. El contenido de la evaluación se refiere a varias fuentes de información

6. Si el alumno reclama puede conseguir

7. Me dan razones de mis aciertos y mis errores 
Tras ver el porcentaje de alumnos que ha utilizado como respuesta cada una de las tres categorías con que pueden hacerlo en cada caso y traducidos esos datos numéricos a su significado cualitativo podríamos realizar las siguientes observaciones. Los alumnos consideran que los exámenes al uso, fomentan el memorismo en mucha mayor medida que la elaboración personal y creativa de los contenidos.

Igualmente entienden que se pone mayor énfasis en aspectos teóricos que en la aplicación práctica en esos exámenes. Este hecho provoca una gran contradicción, ya que se supone que en nuestros centros han de fomentarse, entre otras cuestiones, la capacidad científica y pedagógica.

Según la opinión del alumnado, el profesor, en cierta medida (casi un 40,0\% de las respuestas), muestra a través del examen, algunos rasgos autoritarios o trata de manifestar un poder que en realidad nada tiene que ver con lo que ha de ser la evaluación desde un punto de vista pedagógico.

Habitualmente los exámenes se realizan tomando como punto de referencia varias fuentes de información. Esta suele ir desde los propios apuntes del profesor, hasta determinados textos propuestos por éste. No obstante los apuntes del profesor ocupan un lugar importante como contenidos a evaluar, lo cual, lamentablemente sintoniza en gran medida con lo ya observado en este mismo estudio, en lo referente a la metodología y evaluación que se sigue en nuestros centros.

En cuanto a la nota que el profesor pone, parece ser, a juzgar por la distribución de los porcentajes observada, que éste, aunque no de manera clara, se muestra accesible y con cierta predisposición a la modificación, si procede, de la misma lo que viene a mostrar cómo en cierto modo el distanciamiento entre profesores y alumnos en ocasiones se da y es algo mayor de lo que sería deseable.

Por último, sucede algo parecido con la penúltima de las dimensiones planteadas anteriormente. Efectivamente, aunque en esta ocasión el porcentaje de profesores que explican a sus alumnos en qué ha acertado o errado es superior al observado en el punto anterior, éste no llega a ser lo suficientemente alto como para convenir en que éste hecho sea absolutamente habitual y se de con total frecuencia, lo cual redunda nuevamente en las cuestiones planteadas varias veces en este mismo epígrafe.

\section{8. La opinión del alumno en los sistemas de evaluación empleados por los profesores}

A continuación vamos a tratar de saber si es tenida en cuenta la opinión del alumnado en los distintos sistemas de evaluación que se utilizan en nuestros centros.

Para ello les hemos planteado una pregunta de respuesta simple en los siguientes términos: "Dígame si los profesores tienen en cuenta su opinión sobre el tipo de evaluación que suelen aplicar". Estas han sido sus respuestas. 


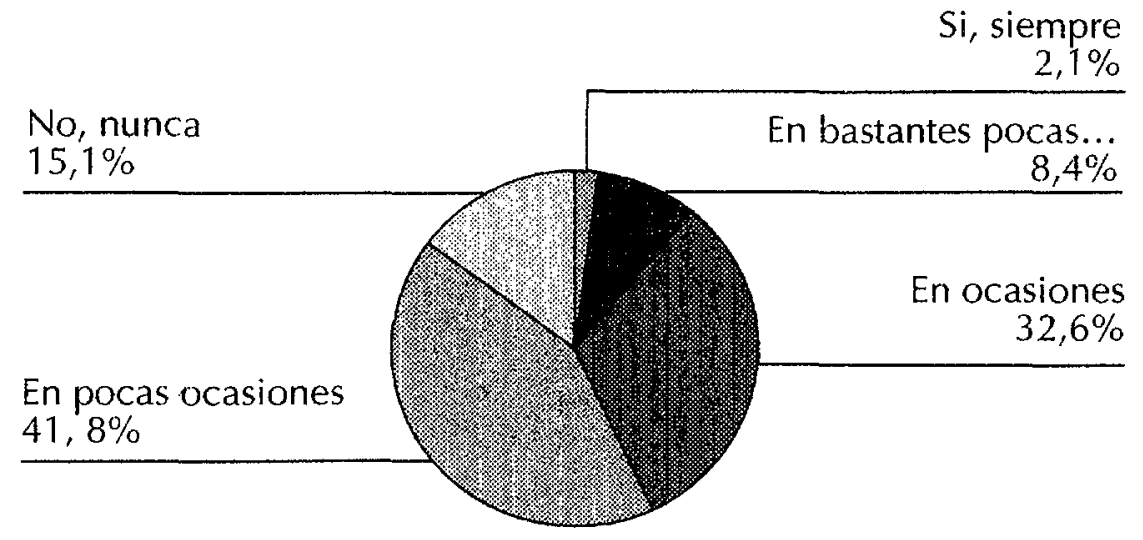

Gráfico n‥ 2. La opinión del alumno en los sistemas de evaluación empleados por los profesores

La distribución de los porcentajes de las respuestas a esta cuestión nos indica que la opción "en pocas ocasiones" es la que alcanza un más alto porcentaje de respuestas.

Además, si extrapolamos los porcentajes de la opción intermedia "en ocasiones", y comparamos la suma de los de las opciones extremas; las dos primeras, que indican cierta asiduidad en esta práctica y las dos últimas, que denotan escasa frecuencia de este hecho, observaremos que se queda en un muy considerable 56,9\% de las últimas frente a un paupérrimo $10,5 \%$ de las primeras con lo que forzosamente hemos de concluir que el profesorado de nuestros centros apenas cuenta con el alumnado cuando se trata de ver qué opina sobre el plan de evaluación empleado.

\section{La satisfacción entre los estudiantes de Educación Física}

Bajo este epígrafe, vamos a tratar de recoger el grado de satisfacción que muestran los estudiantes con respecto a la actividad docente realizada por sus profesores mediante una pregunta estimatoria planteada de la siguiente manera: ¿Estas satisfecho con la actividad docente que desarrollan tus profesores?

Los datos porcentuales que posteriormente analizaremos, son los que mostramos a continuación:

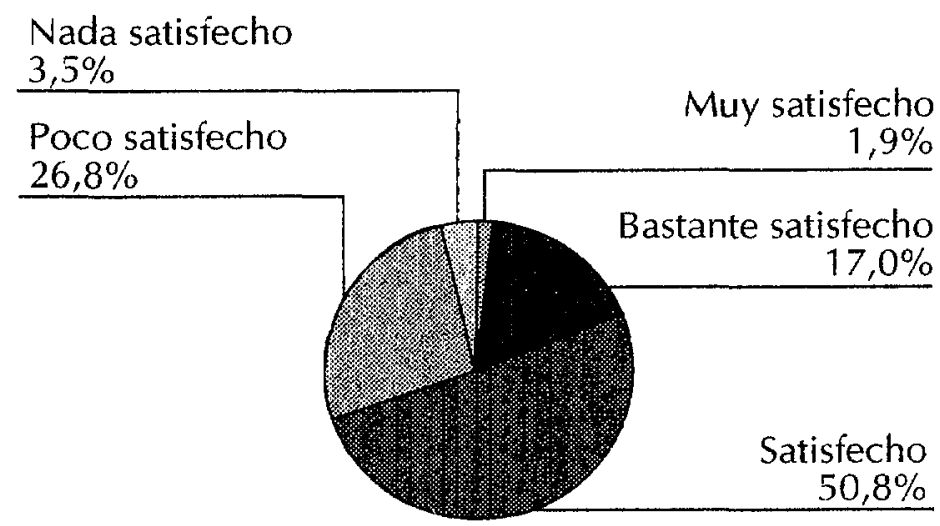

Gráfico no. 3. Grado de satisfacción obtenido en los estudios por nuestro alumnado 
En términos generales, la mayor proporción de respuestas, un 50,8\%, corresponde a la opción de "satisfecho", que concretamente contabiliza 453 respuestas y aparece además claramente diferenciado del resto de las opciones. Por otro lado, si a esta opción le sumamos los porcentajes obtenidos por "bastante satisfecho" $(17,0 \%)$ y "muy satisfecho" (1,9\%), nos encontramos con que al menos un 69,7\% del alumnado, es decir casi tres cuartas partes del mismo encuentra satisfacción, en un grado $u$ otro, en la actividad docente que desarrollan sus profesores, lo cual indudablemente, supone un porcentaje muy considerable que, entre otras cosas que y pese a todo to apuntado hasta ahora por nuestros alumnos en apartados anteriores, muestra la existencia de una conexión importante entre los intereses de los alumnos y lo que reciben en sus respectivos centros.

Ahora bien, tampoco conviene perder de vista que el nivel de insatisfacción, sumadas las opciones "poco satisfecho" $(26,8 \%)$ y "nada satisfecho" (3,5\%), supone un también importante $30,3 \%$ del alumnado, es decir que algo más de una cuarta parte del mismo. Esto pone de manifiesto que, a pesar de las cifras observadas, todavía queda mucho camino por recorrer hasta alcanzar unos niveles de satisfacción óptimos entre nuestro alumnado.

\section{Conclusiones y propuestas}

- A pesar de todo lo que en éste y en otros estudios se ha comentado sobre la lección magistral y aunque esta opción metodológica es la que cuenta con menos aceptación entre nuestros alumnos, ésta sigue siendo la forma de enseñanzaaprendizaje más utilizada en nuestros centros, seguida de las "clases teóricoprácticas" y las "clases prácticas", por lo que proponemos que se den los pasos necesarios para poder superar esta ya secular tendencia didáctica, buscando nuevas fórmulas que hagan más gratificante al alumnado nuestra labor pedagógica. En el lado opuesto, es decir en el de las formas menos utilizadas, se encuentran los "seminarios", "el estudio de casos", "los grupos de discusión" y "la lección impartida por el alumno", fórmulas en las que el alumnado participa de forma más "activa" y que por tanto habría que potenciar.

- Por otra parte entre las opciones metodológicas que cuentan con mayor aceptación entre el alumnado, se hallan las "clases prácticas", decantándose claramente por ella como forma "muy adecuada" de enseñanza-aprendizaje, por lo que parece igualmente prudente la conveniencia de su apoyo. En el extremo opuesto, se halla la "lección magistral", que como opción metodológica utilizada en nuestros centros, tiene escasa aceptación entre el alumnado y sobre la que ya hemos comentado que convendría llevar a cabo alguna reflexión.

- Entre los sistemas de evaluación más utilizados en nuestros centros, no hay duda de que el "examen escrito" constituye la fórmula mayoritariamente utilizada, tal y como indica casi la totalidad de nuestros alumnos, seguida por los "trabajos colectivos" y los "trabajos individuales". Por contra, entre los menos utilizados se hallan los "supuestos prácticos" y especialmente los "exámenes orales", que con $92,8 \%$ de respuestas "poco utilizado", queda claro que es la fórmula menos utilizada. 
De todos estos sistemas de evaluación citados, "los supuestos prácticos" es la alternativa que cuenta con mayor aceptación entre nuestros alumnos, tras ellos aparecen los "trabajos colectivos" y los "trabajos individuales", aunque el nivel de aceptación de estos no está muy claro que sea especialmente elevado. Del resto de las opciones formuladas, destaca como poco aceptada la de los "exámenes orales" ya que así la califican el alumnado con casi un 50,0\% de las respuestas computadas.

Por su parte el "examen escrito", que anteriormente aparecía como el sistema de evaluación más utilizado, aparece ahora considerado como poco adecuado, con lo que nuevamente puede hablarse de cierto desfase entre lo que el alumnado se encuentra en sus centro y lo que realmente parece demandar, desfase que convendría subsanar cuanto antes.

No obstante, tratando de hacer especial hincapié en este punto, entendemos que parece congruente pensar que el tipo de evaluación que más o menos se configura como predominante, refuerza la metodología que en apartados anteriores contemplábamos también como predominante y viceversa.

- Todo lo apuntado puede considerarse hasta cierto punto como una consecuencia lógica de lo apuntado en los apartados 3. 3. y 3. 8. de este estudio, en los que se indica que los profesores apenas cuentan con la opinión de sus alumnos ni a la hora de llevar a cabo el desarrollo de sus asignaturas, ni a la hora de optar por un sistema u otro de evaluación, hecho éste que tendrá que ser sometido al oportuno estudio con el fin de reconducirlo, si ello es posible, hacia derroteros más acordes con las demandas de nuestro alumnado.

- En definitiva, con respecto a cuestiones de Metodología y Evaluación en nuestros centros, hay que significar que las personas protagonistas del proceso de enseñanza-aprendizaje saben lo que quieren y que se percibe con honda preocupación, un claro desfase entre lo que demanda nuestro alumnado y lo que les estamos ofreciendo, entre la praxis cotidiana y los deseos de los alumnos, por lo que entendemos que convendría realizar una seria reflexión al respecto que permitiera acceder a conceptos y métodos de aprendizaje y evaluación renovados, más ricos, humanizados e integrales.

- En cuanto al grado de satisfacción con la actividad desarrollada en sus centros de estudio, puede afirmarse que si bien cabe considerarlo como mayoritario, puesto que el 69,7\% manifiesta distintos niveles de satisfacción (especialmente destaca el 50,8 de alumnos que dicen estar satisfechos), también existe un considerable $30,3 \%$ de nuestros alumnos se muestran poco o nada satisfechos. En todo caso, entendemos que existe cierto nivel de contradicción entre lo manifestado por los alumnos en otros epígrafes de este estudio y lo que éstos indican en este apartado, contradicción que sería importante detectar en posteriores estudios a qué se debe.

- Para finalizar, cabe indicar, que las conclusiones extraídas de este estudio, no pueden considerarse, en modo alguno, definitivas sino como hipótesis que nos llevan a pensar que este trabajo debería continuarse en el futuro introduciendo otros elementos de juicio que permitan ahondar en el porqué de los resultados obtenidos. 


\section{Bibliografía}

CANDAU, V. M. (1987) La didáctica en cuestión: Investigación y enseñanza. Madrid: Narcea

CONTRERAS DOMINGO, J. (1991) Enseñanza, curriculum y profesorado. Madrid: Akal Universitaria.

ESCUDERO, J.M. (1986) Innovación e investigación educativa. Revista de información e investigación educativa $n^{2} 1$

GIMENO SACRISTAN, J. (1988) El curriculum: una reflexión sobre la práctica. Madrid: Morata.

IBERNON, F. (1989) La formación del profesorado. Barcelona. Laia

LOZA OLAVE, E. (1997) La especialidad de Educucación Física en las E.U.M y/o Facultades de Educación. Tesis doctoral no publicada. Zaragoza

MARCELO, C. (1987) El pensamiento del profesor. Barcelona: CEAC.

UBIETO, A. (1978) Cómo se programa un tema o una Unidad Didáctica.

Zaragoza: I. C. E. Universidad de Zaragoza

VILLAR ÁNGULO, L.M. (1990) El profesor como profesional: Formación y desarrollo personal. Granada: Servicio de Publicaciones de la Universidad de Granada. 